\title{
A Novel Method for Psychiatric Clinical Diagnosis In Schizophrenic Symptoms Detection
}

\author{
Yan Zhang ${ }^{1 *}$, Jianguo Shi ${ }^{1,2,3}$, Feihu Liu $^{1,2,3}$, Zunxiao Dai ${ }^{1,2}$, Bin $W_{u^{1,2}}$, Qiangju $\mathbf{W u}^{1,2}$, Xiaohua Cui ${ }^{1,2}$, \\ Xiangnong $\mathrm{Du}^{1,2}$, Lisha Sun ${ }^{1,2}$ and Tanghui $\mathrm{Xu}^{4}$ \\ ${ }^{1}$ Xi'an Mental Health center, Xi'an 710100, Shaanxi, China
}

${ }^{2}$ The Affiliated Mental Health Institute of Xi'an School of Medicine, Xi'an 710061, Shaanxi, China

${ }^{4}$ The Affiliated NO. 1 hospital of Xi'an School of Medicine, Xi'an 710000, Shaanxi, China

${ }^{3}$ Dr Jianguo Shi, Dr Feihu Liu contributed equally to this article as co-first author

Received: September 21, 2017; Published: October 02, 2017

*Corresponding author: Yan Zhang, Xi'an Mental Health center, The eastern section of Hangtian street, Xi'an 710100, Shaanxi, China. Tel : +86-029-63609232. Email : zhan_777@163.com

\begin{abstract}
Study was aim to evaluate reliability and psychiatric clinical value of Quantum Resonance Spectrometer (QRS) on schizophrenia symptoms detection. There were 100 schizophrenic patients and 100 normal controls in study. Symptoms of subjects respectively obtained from QRS test and psychiatrists' diagnoses. The results of two methods were compared. Also symptoms of schizophrenia patient and 100 normal controls were discriminated using QRS test. Kappa value of symptoms detection had good consistency with psychiatrists' diagnose. There were 7 symptoms more than 0.60 in total 11 symptoms of schizophrenic and 7 symptoms detection results of AUC of ROC more than 0.70 . The same consistency was also being found in Youden index. In this study, Hallucination, Delusions, Stereotypy, Virbigeration, Pathological indolent, Psychomotor retardation, and Psychomotor excitement total 7 symptoms were be predicted by QRS. They have good consistency with psychiatrists' diagnoses. QRS in schizophrenia symptoms detection have a predictable value for outcome, is an objective identification and diagnosis instrument, and may promote psychiatric clinical diagnosis development.
\end{abstract}

Keywords : Quantum Resonance Spectrometer (QRS); Symptom; Schizophrenic; Detection; Diagnoses

Abbreviations : QRS: Quantum Resonance Spectrometer; CSF: Cerebrospiral Fluid; CT: Computed Tomography; EDA: Electro Dermal Activity

\section{Introduction}

Schizophrenia is one of the most severe and chronic forms of mental illness. The current psychiatric classification systems, the International Statistical Classification of Diseases and Related Health Problems $10^{\text {th }}$ Revision (ICD-10) [1] WHO, and the Diagnostic and Statistical Manual of Mental Disorders $4^{\text {th }}$ Edition (DSM-IV) [2], base the diagnosis of schizophrenia exclusively on its clinical symptomatology. We have studied quantum resonance of schizophrenics and normal people while subjects freely holding the sensor. This method is called exploratory quantum resonance test. Therefore, the above physiological or neuroscience defects, the change of electromagnetic wave signals $[3,4]$ or yin-yang meridian, may show promise as biological markers of schizophrenia $[5,6]$. The aim of the research was to develop an easily available and replicable biological objective system, Quantum Resonance Spectrometer (QRS), which based on yin-yang meridian, could be used as predictor of psychiatric diagnoses, and could attenuate subjective ratings [7].
In our previous studies, only psychiatric cardinal symptoms have revealed consistent distribution as diagnosis of psychiatrist [8-13]. Based on these findings, we have proposed that the QRS test may be useful as a biological marker for the clinical diagnosis of psychiatric disorders [8-13]. In this study, we aim to check QRS performance on schizophrenic symptoms between schizophrenics and non schizophrenics. According to results of the consistency analysis, we examined the possibility of QRS on clinical diagnosis of schizophrenic.

\section{Material and Methods}

\section{Subjects}

We studied 100 schizophrenic patients and 100 normal controls. The patients were in/outpatients recruited from the Mental Health center of Xi'an (Xi'an, PR China). Diagnoses were made by experienced psychiatrists according to the ICD-10 criteria for research [1] WHO. The control subjects were also recruited from 
the Mental Health center of Xi'an. Most controls were employees of these hospitals. Table 1 shows the demographic characteristics of the subjects. There were no significant differences between the groups in age, and gender, except duration of illness. Psychiatric patients who had a history of alcohol abuse or illicit substance abuse, or head injury were excluded from the study; also excluded were those with convulsive, neurologic or ophthalmologic disorders. The normal controls were healthy volunteers without physical, ophthalmologic, neurological or psychiatric disorders, and there was no family history of psychiatric disorders as distant as third degree relatives. This study was approved by the Ethics Committees of the Mental Health center of Xi'an. Written informed consent was obtained from all participants, after the procedures and possible risks of the study were fully explained.

Table 1: Clinical and demographic characteristics of the subjects.

\begin{tabular}{|c|c|c|}
\hline Diagnosis & Schizophrenia & Control \\
\hline Subjects(n) & 100 & 100 \\
\hline Age (years, mean SD)a & $38.5 \pm 13.6$ & $41.9 \pm 12.3$ \\
\hline Gender (M/F)b & $46 / 54$ & $44 / 56$ \\
\hline $\begin{array}{c}\text { Duration of illness } \\
\text { (years, mean SD) b }\end{array}$ & $11.1 \pm 10.1$ & \\
\hline
\end{tabular}

aT-Test; $\mathrm{t}=0.72, \mathrm{P}=0.458, P>0.05$

${ }^{\mathrm{b}}$ T-Test; $\mathrm{t}=0.4574 \mathrm{df}=1167, P>0.05$

\section{Procedure}

For this study, we explored and developed a biological wave sensing device, a QRS (TJQ-D, Chongqing, China), in psychiatric examination. It comprised a biological wave detection unit, a biological operation control unit, a power, and a follower, wherein, a contact of the biological operation control unit is connected with an input end of the follower, and the other contact is connected with an cathode of the power, the biological wave detection unit is made of conductors, and connected with the cathode of the power, or the input end of the follower. This system automatically recorded the special biological wave and could effectively transform message into electrical signals which can be dealt by computers, to get resonance score.

The subject sat on a chair and was freely holding the sensor in his/her hand. The special biological wave of subject was collected and transformed electrical signals compared with computer control data. Then the resonance score were obtained by computer. Measurement and analysis released by the brain the size of the vibration frequency (a weak magnetic field fluctuations in energy), to determine whether there is a psychiatric symptoms, score $\leq-6$ as normal values, -7 as mildly abnormal, -8 as moderate abnormalities, -9 or more as severe abnormalities, and compared with equipment standards set by the magnetic field to distinguish between normal or abnormal on thought disorder. In this study, all human subjects have been approved by the Xi'an Institute of Mental Health ethics committee and have therefore been performed in accordance with the ethical standards laid down in the 1964 Declaration of Helsinki and its later amendments.

\section{Statistical analysis}

As mentioned above, there were no significant differences between the groups in the demographic data gender and duration of illness, (Table 1). Thus, concordance for each thought disorder symptoms were tested by Kappa analysis, Youden index and ROC curve compute with no covariate. Kappa was characterized values < 0 as indicating no agreement and $0 \sim 0.20$ as slight, $0.21 \sim 0.40$ as fair, $0.41 \sim 0.60$ as moderate, $0.61 \sim 0.80$ as substantial, and $0.81 \sim 1$ as almost perfect agreement [14]. The AUC of ROC curve was equal to the probability that a classifier will rank a randomly chosen positive instance higher than a randomly chosen negative one [15], and was characterized values $<0.5$ as indicating no diagnostic practical situation and $0.50 \sim 0.70$ as low diagnostic value, $0.71 \sim 0.90$ as moderate, $>0.91$ as higher diagnostic value. For pair wise multiple comparisons, Bonferroni adjustment was used (SPSS manual). Statistical significance was set at $\mathrm{P}<0.01$. All statistical analyses were performed using SPSS for Windows version 17.0.

\section{Results}

\section{Sensitivity and specificity of QRS detection on schizophrenic symptoms}

To evaluate QRS performance on schizophrenic symptoms, we studied 11 symptoms of 100 schizophrenic patients and 100 control subjects. 11 symptoms were made by experienced psychiatrists according to the ICD-10 criteria. Diagnose of one symptom was characterized 1 as there was the symptom, 0 as there was no symptom. On the other side, QRS data were collected by researchers who did not know the result of diagnoses. Sensitivity and specificity of QRS detection were shown (Table 2).

Table 2 : Sensitivity and specificity of QRS performance on schizophrenic symptoms $(n=200)$.

\begin{tabular}{|c|c|c|c|c|}
\hline \multirow[b]{2}{*}{ Symptoms } & \multicolumn{2}{|c|}{ Sensitivity } & \multicolumn{2}{|c|}{ Specificity } \\
\hline & Mean & $\begin{array}{c}\text { Confidence interval } \\
(95 \%)\end{array}$ & Mean & $\begin{array}{c}\text { Confidence interval } \\
(95 \%)\end{array}$ \\
\hline Hallucination & 0.976 & $0.945 \sim 1.000$ & 0.701 & $0.648 \sim 0.756$ \\
\hline Delusions & 0.965 & $0.918 \sim 1.000$ & 0.710 & $0.627 \sim 0.793$ \\
\hline Stereotypy & 0.894 & $0.796 \sim 0.992$ & 0.854 & $0.767 \sim 0.942$ \\
\hline Compulsions & 0.500 & $0.217 \sim 0.782$ & 0.840 & $0.764 \sim 0.917$ \\
\hline Stereotyped speech & 0.611 & $0.385 \sim 0.836$ & 0.951 & $0.904 \sim 0.997$ \\
\hline Virbigeration & 0.714 & $0.477 \sim 0.950$ & 0.941 & $0.892 \sim 0.991$ \\
\hline Pathological indolent & 0.918 & $0.860 \sim 0.976$ & 0.250 & $0.174 \sim 0.674$ \\
\hline
\end{tabular}




\begin{tabular}{|c|c|c|c|c|}
\hline Pathological disobey & 0.736 & $0.538 \sim 0.934$ & 0.887 & 0.891 \\
\hline Psychomotor retardation & 0.888 & $0.805 \sim 0.972$ & 0.854 & $0.818 \sim 0.956$ \\
\hline Psychomotor excitement & 0.894 & $0.879 \sim 1.000$ & 0.575 & $0.849 \sim 0.986$ \\
\hline Lack of insight & 0.968 & $0.932 \sim 1.000$ & \multicolumn{2}{|c|}{ Specificity } \\
\hline \multirow{2}{*}{ Symptoms } & \multicolumn{2}{|c|}{ Sensitivity } & Mean & Confidence interval \\
\cline { 2 - 5 } & Mean & Confidence interval & (95\%) & $0.481 \sim 0.669$ \\
\hline Hallucination & 0.976 & $0.945 \sim 1.000$ & 0.701 & $0.627 \sim 0.793$ \\
\hline Delusions & 0.965 & $0.918 \sim 1.000$ & 0.710 & $0.767 \sim 0.942$ \\
\hline Stereotypy & 0.894 & $0.796 \sim 0.992$ & 0.854 & $0.764 \sim 0.917$ \\
\hline Compulsions & 0.500 & $0.217 \sim 0.782$ & 0.840 & $0.904 \sim 0.997$ \\
\hline Stereotyped speech & 0.611 & $0.385 \sim 0.836$ & 0.951 & $0.892 \sim 0.991$ \\
\hline Virbigeration & 0.714 & $0.477 \sim 0.950$ & 0.941 & $0.174 \sim 0.674$ \\
\hline Pathological indolent & 0.918 & $0.860 \sim 0.976$ & 0.250 & $0.818 \sim 0.956$ \\
\hline Pathological disobey & 0.736 & $0.538 \sim 0.934$ & 0.887 & $0.801 \sim 0.981$ \\
\hline Psychomotor retardation & 0.888 & $0.805 \sim 0.972$ & 0.891 & $0.849 \sim 0.986$ \\
\hline Psychomotor excitement & 0.894 & $0.879 \sim 1.000$ & 0.854 & $0.481 \sim 0.669$ \\
\hline Lack of insight & 0.968 & $0.932 \sim 1.000$ & 0.575 & \\
\hline
\end{tabular}

Predictive ability of QRS detection on schizophrenic symptoms

To evaluate predictive ability of QRS performance on schizophrenic symptoms, we used positive predictive value and negative predictive value. Results were shown (Table 3 ).

Table 3 : Sensitivity and specificity of QRS performance on schizophrenic symptoms $(\mathrm{n}=200)$.

\begin{tabular}{|c|c|c|c|c|}
\hline \multirow{2}{*}{ Symptoms } & \multicolumn{2}{|c|}{ Positive predictive value } & \multicolumn{2}{|c|}{$\begin{array}{c}\text { Negative predictive } \\
\text { value }\end{array}$} \\
\cline { 2 - 5 } & Mean & $\begin{array}{c}\text { Confidence } \\
\text { interval } \\
\mathbf{( 9 5 \% )}\end{array}$ & Mean & $\begin{array}{c}\text { Confidence } \\
\text { interval } \\
\text { (95\%) }\end{array}$ \\
\hline Hallucination & 0.711 & $0.630 \sim 0.794$ & 0.975 & $0.942 \sim 1.000$ \\
\hline Delusions & 0.715 & $0.633 \sim 0.797$ & 0.964 & $0.568 \sim 1.000$ \\
\hline Stereotypy & 0.790 & $0.669 \sim 0.912$ & 0.929 & $0.863 \sim 0.996$ \\
\hline Compulsions & 0.300 & $0.099 \sim 0.500$ & 0.925 & $0.867 \sim 0.982$ \\
\hline $\begin{array}{c}\text { Stereotyped } \\
\text { speech }\end{array}$ & 0.733 & $0.509 \sim 0.956$ & 0.917 & $0.859 \sim 0.976$ \\
\hline Virbigeration & 0.666 & $0.428 \sim 0.905$ & 0.952 & $0.908 \sim 0.997$ \\
\hline $\begin{array}{c}\text { Pathological } \\
\text { indolent }\end{array}$ & 0.963 & $0.923 \sim 1.000$ & 0.125 & $0.104 \sim 1.000$ \\
\hline $\begin{array}{c}\text { Pathological } \\
\text { disobey }\end{array}$ & 0.608 & $0.407 \sim 0.805$ & 0.934 & $0.878 \sim 0.989$ \\
\hline $\begin{array}{c}\text { Psychomotor } \\
\text { retardation }\end{array}$ & 0.905 & $0.827 \sim 0.984$ & 0.872 & $0.777 \sim 0.967$ \\
\hline $\begin{array}{c}\text { Psychomotor } \\
\text { excitement }\end{array}$ & 0.790 & $0.783 \sim 0.978$ & 0.929 & $0.918 \sim 1.000$ \\
\hline Lack of insight & 0.669 & $0.590 \sim 0.748$ & 0.953 & $0.901 \sim 1.000$ \\
\hline
\end{tabular}

\section{Consistency of QRS detection and psychiatrists diagnoses}

Consistencies of examined symptoms were judged by Kappa analysis, Youden index and ROC, listed in the (Table 4). Psychiatrists Diagnose was characterized 1 as there was one symptom. The higher the score values mean the more symptoms. But QRS Score ( $\leq-6$ as normal values, -7 as mildly abnormal, -8 as moderate abnormalities, -9 or more as severe abnormalities) was that the smaller the score values mean the more symptoms. Total score of schizophrenic disorders by QRS detection and psychiatrists' diagnoses in schizophrenia and controls were also with good consistency.

\section{Discussion}

Traditional clinical diagnosis of schizophrenia is based on patient interviews and observation of the patient's behavioral patterns, is subjective. More and more instruments [16] began to consider used in the diagnosis of psychiatric disorders with the progress of science and technology, which would be more objective and controlled. Schizophrenic symptoms may be based on a neurobiological brain dysfunction. The need for more precise and reliable diagnostic markers is underscored by a well established association between the duration of untreated psychosis and an unfavorable clinical outcome of schizophrenia [17-19]. Therefore, in addition to the interview and observation of the patient, a biological marker related to the brain dysfunction or brain potentials of schizophrenia may also be useful in determining the clinical diagnosis of schizophrenia [4,16,20,21].

In order to find a biological marker of schizophrenia, many researchers have performed psycho physiological or cognitive neuroscience tests related to the potential brain dysfunction of schizophrenia [19,22]. During a 20 year period, the method including Symptomatology, Cerebrospiral fluid (CSF) biochemistry, Computed Tomography (CT) scan, neurophysiologic and psycho physiologic (Electro Dermal Activity, EDA) parameters before antipsychotic treatment was initiated $[19,23]$. At the same time human body is an aggregate of numerous cells, which continuously grow develop, split, regenerate and die. In the process of cellular split-up and renewal, the charged bodies of nucleus and extra 
nuclear electrons as the basis unit of a cell are moving and changing ceaselessly at a high speed as well, emitting electromagnetic waves without interruption. The signals of electromagnetic waves emitted by human bodies represent the specific condition of human body and therefore, different signals of electromagnetic waves will be emitted by the conditions of good health, sub-health, diseases, etc $[24,25]$. The conditions of life can be analyzed if such specific electromagnetic wave signals can be analyzed [26,27]. The electro dermal tests also be tried to evaluate the diagnostic accuracy of in allergic subjects $[23,28]$.

Therefore, we hypothesized that the QRS test may be specific to schizophrenia. However, the samples used in our previous studies were not including controls. We devoted a substantial amount of time to performing the test and analyzing the data [10-13]. Now we started to study related-symptom of psychiatric disorders. Consequently, we did the first schizophrenic sample study to confirm our previous findings. Thus, in addition to the above physiological or neuroscience defects, QRS disorder may also be a biological marker of schizophrenia.

As a result, we obtained the following high checking rate symptoms: Hallucination, Delusions, Stereotypy, Virbigeration, Pathological indolent, psychomotor retardation, and psychomotor excitement total 7. Kappa value of above 7 symptoms are $>0.61$, and AUC of ROC are $>0.71$. These symptoms were all higher value in (Table 4). Some of them almost perfect agreement with psychiatrists' diagnoses. But the problem could be found in stereotyped speech whose Kappa value is 0.601 , but its sensitivity, specificity, positive predictive value, negative predictive value are high. This symptom might exist in normal persons. Or it might be too sensitivity. Fortunately Kappa coefficient and the Youden value could show the same meaning. Stereotyped speech's detection of QRS probably more effective was after debugging.

Table 4: Consistency of QRS detection and psychiatrists' diagnoses on schizophrenic symptoms $(\mathrm{n}=200)$.

\begin{tabular}{|c|c|c|c|c|c|}
\hline \multirow{2}{*}{ Symptoms } & \multirow{2}{*}{ Kappa value } & Youden index & AUC of ROC \\
\cline { 2 - 6 } & & Mean & $\begin{array}{c}\text { Confidence interval } \\
\text { (95\%) }\end{array}$ & $\begin{array}{c}\text { Mean } \\
\text { Confidence interval } \\
\text { (95\%) }\end{array}$ \\
\hline Hallucination & 0.649 & 0.677 & $0.589 \sim 0.768$ & 0.925 & $0.890 \sim 0.960$ \\
\hline Delusions & 0.648 & 0.675 & $0.585 \sim 0.767$ & 0.904 & $0.860 \sim 0.948$ \\
\hline Stereotypy & 0.731 & 0.748 & $0.618 \sim 0.880$ & 0.891 & $0.818 \sim 0.964$ \\
\hline Compulsions & 0.265 & 0.340 & $0.046 \sim 0.542$ & 0.678 & $0.484 \sim 0.872$ \\
\hline Stereotyped speech & 0.601 & 0.562 & $0.332 \sim 0.792$ & 0.867 & $0.750 \sim 0.984$ \\
\hline Virbigeration & 0.637 & 0.655 & $0.414 \sim 0.897$ & 0.869 & $0.744 \sim 0.993$ \\
\hline Pathological indolent & 0.629 & 0.168 & $0.262 \sim 0.594$ & 0.815 & $0.874 \sim 0.998$ \\
\hline Pathological disobey & 0.578 & 0.623 & $0.414 \sim 0.834$ & 0.930 & $0.695 \sim 0.936$ \\
\hline $\begin{array}{c}\text { Psychomotor } \\
\text { retardation }\end{array}$ & 0.779 & 0.779 & $0.657 \sim 0.903$ & 0.924 & $0.876 \sim 0.985$ \\
\hline $\begin{array}{c}\text { Psychomotor } \\
\text { excitement }\end{array}$ & 0.731 & 0.748 & $0.769 \sim 0.964$ & $0.963 \sim 0.985$ \\
\hline Lack of insight & 0.530 & 0.543 & $0.443 \sim 0.644$ & 0.932 & $0.895 \sim 0.969$ \\
\hline
\end{tabular}

There were always doubts on a new method development [29]. We need to be more professionals; pay more attention to details and spend more time to research the new idea. May be we still doubt after run a long way. So, we studied 100 schizophrenic patients. There were 100 controls in analysis that mainly aim to equal with the schizophrenic. In all the used examples of symptom, the optimum discriminator is QRS score obtained in Hallucination, Delusions, Stereotypy, Virbigeration, Pathological indolent, Psychomotor retardation, and Psychomotor excitement. The results indicate that Kappa coefficient, sensitivity, specificity, positive predictive value, negative predictive value and the Youden value of QRS in above7 symptoms may be of predictive value and are compatible with psychiatrists' diagnoses of schizophrenia.

Jianguo Shi et al. [12] performed also tried to discriminate 37 hyperthymic from 58 depressed patients and 27 healthy controls using QRS data, and obtained a high rate of discrimination with both the sensitivity being over 70\%. Zhifang Guo et al. [10] also tried to evaluate detection of the hallucination symptom in 241 schizophrenics using QRS. The auditory hallucination symptom was detected by QRS with a sensitivity of approximately $98 \%$ and an accuracy of approximately $90 \%$. These results suggest that QRS may be useful for clinical diagnosis of schizophrenic. These clinical symptoms include positive symptoms, negative symptoms and social withdrawal. Presently, this phenomenological diagnostic approach is without an alternative due to a lack of clear biological markers for the disorder. The need for more precise and reliable diagnostic markers is underscored by a well established association between the symptom detection of QRS and psychiatric disorders. QRS is an objective diagnosis instrument, and could promote psychiatric clinical diagnosis development. QRS in schizophrenic symptoms detection possess higher authenticity, consistency higher, and the high diagnostic value.

In summary, detection of QRS in schizophrenic 11 symptoms seem to have a predictable value for outcome in schizophrenia, early identification of them might be a challenge for our future treatment strategies. QRS might become an objective diagnosis instrument, could attenuate psychiatrists' subjective ratings [7], and could promote psychiatric clinical diagnosis development. 


\section{Conflict of Interest}

The authors declare no conflicts of interest.

\section{Contributors}

Mr. Jianguo Shi designed the study. Yan Zhang wrote the analysis plan, extracted and cleaned the data. Feihu Liu performed the data analysis, with input and data interpretation from all authors. Mr. Shi and Yan Zhang wrote the first draft of the manuscript and all authors commented extensively and approved the final draft.

\section{Acknowledgment}

The current study is supported by Xi'an science and technology projects fund (HM1122(1) and SF1515(3)), and projects fund of Xi'an municipal health and Family Planning Commission (J201602034(II)), for which the corresponding authors Yan Zhang and Jianguo Shi are the principal investigators.

\section{References}

1. Organization WH (1993) The international classification of mental and behavioural disorders: diagnostic criteria for research. WHO, Geneva, Switzerland.

2. APA (2000) Diagnostic and Statistical Manual of Mental Disorders DSMIV-TR Fourth Edition (Text Revision). American Psychiatric Publishing Inc, USA, pp. 1-982.

3. Nilsson BM, Hultman CM, Ekselius L (2009) Test-retest stability of the oral niacin test and electrodermal activity in patients with schizophrenia. Prostag Leukotr Ess 81(5-6): 367-372.

4. Mangina CA, Beuzeron Mangina JH, Grizenko N (2000) Event-related brain potentials, bilateral electrodermal activity and Mangina-Test performance in learning disabled/ADHD pre-adolescents with severe behavioral disorders as compared to age-matched normal controls. Int J Psychophysiol 37(1): 71-85.

5. Jandl M, Steyer J, Kaschka WP (2010) Suicide risk markers in Major Depressive Disorder: A study of Electrodermal Activity and EventRelated Potentials. J Affect Disorders 123(1-3): 138-149.

6. Gruzelier J, Raine A (1994) Bilateral electrodermal activity and cerebral mechanisms in syndromes of schizophrenia and the schizotypal personality. Int J Psychophysiol 16(1): 1-16.

7. Wahlund K, Sorman K, Gavazzeni J, Fischer H, Kristiansson M (2010) Attenuated subjective ratings and skin conductance responses to neutral and negative pictures in non-psychopathic mentally disordered offenders with various diagnoses. Psychiat Res 180(1): 30-34.

8. Yan Zhang, Feihu Liu, Jianguo Shi, Xiaobin Yue, Haitao Zhang, et al. (2014) Exploratory quantum resonance spectrometer as a discriminator for psychiatric affective disorders. J Nerv Ment Dis 202(4): 287-291.

9. Jianguo Shi, Yan Zhang, Feihu Liu, Man Zhang, Xiaobin Yue, et al. (2012) Quantum resonance spectrometer as a discriminator in schizophrenic thought disorder detection. Eur Psychiat 27(1): 1.

10. Yuanyuan Luo, Feihu Liu, Zhifang Guo, Jianguo Shi (2009) Elementary evaluation of detection of the attention disorder and impaired memory via Quantum reaonance spectrometer. Medical Innovation of China 6(17): 41.

11. Zhifang Guo, Jianguo Shi, Feihu Liu, Yuanyuan Luo (2009) Evaluation of common psychiatric symptoms via Quantum Resonance Spectrometer. China Journal of Health Psychology 17(5): 516.
12. Zhifang Guo, Jianguo Shi, Feihu Liu, Yuanyuan Luo (2008) Elementary evaluation of the hallucination symptom via quantum resonance spectrometer. Chin J Behavioral Med Sci 17(6): 537.

13. Jianguo Shi, Yuanyuan Luo, Feihu Liu, Zhifang Guo (2009) Elementary evaluation of detection of the affective disorders via Quantum reaonance spectrometer. Medical Journal of Chiese People's Health 21(8): 797.

14. Landis JR, Koch GG (1977) The Measurement of Observer Agreement for Categorical Data. Biometrics 33(1): 159-174.

15. Fawcett T (2006) An introduction to ROC analysis. Pattern Recogn Lett p. 27.

16. Lencer R, Trillenberg Krecker K, Schwinger E, Arolt V (2003) Schizophrenia spectrum disorders and eye tracking dysfunction in singleton and multiplex schizophrenia families. Schizophr Res 60(1): 33-45.

17. Ceskov E, Drybčák P, Hrobar P, Lorenc M, Procházková H, et al. (2001) The changes of biological markers and treatment efficacy in schizophrenia. Prog Neuro-Psychoph 25(2): 323-335.

18. Oertel-Knöchel V, Bittner RA, Knöchel C, Prvulovic D, Hampel H (2011) Discovery and development of integrative biological markers for schizophrenia. Prog Neurobiol 95(4): 686-702.

19. Lindström LH (1996) Clinical and biological markers for outcome in schizophrenia: A review of a longitudinal follow-up study in Uppsala schizophrenia research project. Neuropsychopharmacol 14(3): 23S-26S.

20. Ceskov E, Drybčák P, Lorenc M (2002) Biological markers and possibilities for predicting therapeutic results in schizophrenia: A methodological contribution. Prog Neuro-Psychoph 26(4): 683-691.

21. Schoen W, Chang JS, Lee U, Bob P, Mashour GA (2011) The temporal organization of functional brain connectivity is abnormal in schizophrenia but does not correlate with symptomatology. Consciousness and Cognition 20(4): 1050-1054.

22. Szymanski S, Kane JM, Lieberman JA (1991) A Selective Review of Biological Markers in Schizophrenia. Schizophrenia Bull 17(1): 99-111.

23. Lewith GT, Kenyon JN, Broomfield J, Prescott P, Goddard J, et al. (2001) Is electrodermal testing as effective as skin prick tests for diagnosing allergies? A double blind, randomised block design study. BMJ. 322(7279): 131-134.

24.Zimmer H, Vossel G, Fröhlich WD (1990) Individual differences in resting heart rate and spontaneous electrodermal activity as predictors of attentional processes: effects on anticipatory heart rate decelaration and task performance. Int J Psychophysiol 1990 8(3): 249-259.

25. Colbert AP, Spaulding K, Larsen A, Ahn AC, Cutro JA (2011) Electrodermal Activity at Acupoints: Literature Review and Recommendations for Reporting Clinical Trials. Journal of Acupuncture and Meridian Studies. 4(1): 5-13.

26. Pontarollo F, Rapacioli G, Bellavite P (2011) Increase of electrodermal activity of heart meridian during physical exercise: The significance of electrical values in acupuncture and diagnostic importance. Complementary Therapies in Clinical Practice 16(3): 149-153.

27. Benedek M, Kaernbach C (2010) A continuous measure of phasic electrodermal activity. J Neurosci Meth 190(1): 80-91.

28. Semizzi M, Senna G, Crivellaro M, Rapacioli G, Passalacqua et al. (2002) A double-blind, placebo-controlled study on the diagnostic accuracy of an electrodermal test in allergic subjects. Clin Exp Allergy. 32(6): 928932.

29. Lewith GT (2003) Can we evaluate electrodermal testing? Complement Ther Med 11(2): 115-117. 


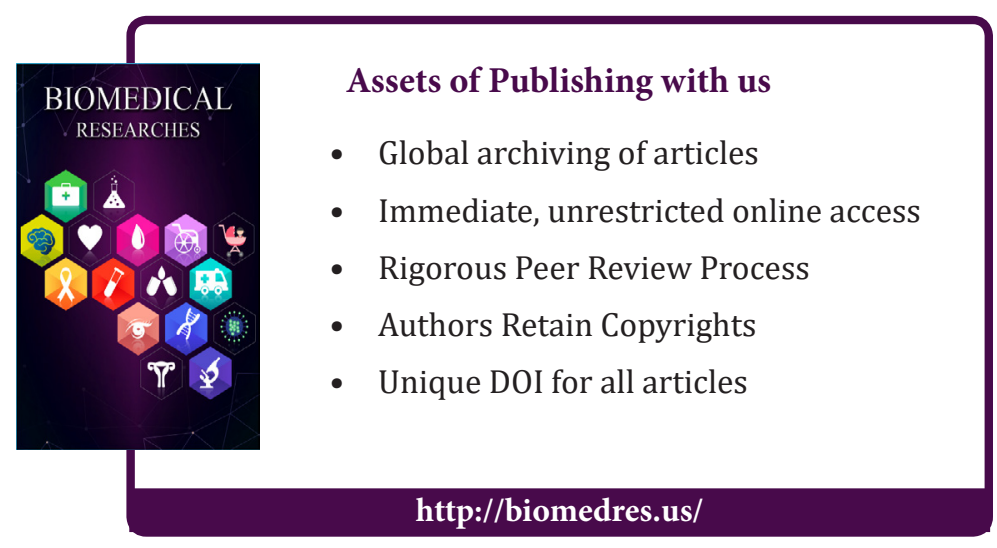

